\title{
Supramolecular assembly by co-crystallization of fumaric acid with 1H-benzotriazole: crystal structure and synthon cooperation
}

\author{
Changfeng Sun ${ }^{1, a}$, Yanyan Pang ${ }^{2, b}$, Ruixin Chen ${ }^{* 2, c}$ \\ ${ }^{1}$ Dongchang College of Liaocheng University, 105 Huanghe Road, Liaocheng \\ Economic Development Zone, Liaocheng, 252000, P. R. China \\ ${ }^{2}$ College of Chemistry and Molecular Engineering, Qingdao University of Science and Technology, \\ Qingdao 266042, P. R. China \\ ascf001@163.com, binorchem1@126.com, ${ }^{\mathrm{c} m a t h c r x @ 126 . c o m ~}$
}

Keywords: Structure characterization; Hydrogen bond; Synthon; Co-crystal

\begin{abstract}
Hydrogen-bonded directed assemblies of one binary co-crystal was validated by single-crystal X-ray diffraction analysis. The supramolecular reaction between symmetric fumaric acid and 1H-benzotriazole produced molecular co-crystal formulated as $\left[\left(\mathrm{C}_{6} \mathrm{H}_{5} \mathrm{~N}_{3}\right) \cdot\left(\mathrm{C}_{4} \mathrm{H}_{4} \mathrm{O}_{4}\right)_{0.5}\right]$ in high yields. Compound contains fumaric acid/1H-benzotriazole in $3: 2$ ratios and displays a 3D (three-dimensional) H-bonded supramolecular structure. The predominant driving force in co-crystal is hydrogen bonds, by which the compound assemble into supramolecular organic framework. With the presence of the reliable hydrogen-bonding donor/acceptor(s) of fumaric acid, the crystalline lattice of co-crystal is managed by $\mathrm{O}-\mathrm{H} \cdots \mathrm{N}$ and $\mathrm{N}-\mathrm{H} \cdots \mathrm{O}$ to afford one-dimensional (1D) acid-base hydrogen-bonding tapes extending along the $\mathrm{b}$ axis direction, which are further extended to distinct 3D arrays via primary synthons (the corresponding $\mathrm{N}-\mathrm{H} \cdots \mathrm{N}$ and $\mathrm{C}-\mathrm{H} \cdots \mathrm{O}$ interactions). This structural study shows that fumaric acid is a supramolecular reagent that can produce well-defined supermolecule in very high yield.
\end{abstract}

\section{Introduction}

Supramolecular organic frameworks are notable representatives in supramolecular chemistry, in which the role of hydrogen bonds (H-bonds), as predominant driving forces, has been well-established. [1-3] Investigation on compounds of organic entities built by paradigms of supramolecular synthons is currently undergoing a burgeoning development taken full advantage of crystal engineering concepts. [4-8] Synthons can be defined that can be used as a guideline in the preparation of particular solid-state structures. The strength of noncovalent interactions ranges from weak, like van der Waals forces, $\mathrm{C}-\mathrm{H} \cdots \mathrm{X}$ hydrogen bonds $(\mathrm{X}=\mathrm{O}, \mathrm{N}),[9-11]$ to strong forces such as $\mathrm{O}-\mathrm{H} \cdots \mathrm{X}$ hydrogen bonds $(\mathrm{X}=\mathrm{O}, \mathrm{N})$. [12] Apart from the strong interactions that are often structure determining, it is the sum of a number of different weak forces which leads to the observed solid state structure. [13] This was demonstrated by Jones et al. with the molecular complexes of phenazine with various dicarboxylic acids. [14] Other systems that utilize $\mathrm{O}-\mathrm{H} \cdots \mathrm{O}$ and $\mathrm{C}-\mathrm{H} \cdots \mathrm{O}$ interactions with carboxylic acids [15] or $\mathrm{O}-\mathrm{H} \cdots \mathrm{N}$ and $\mathrm{C}-\mathrm{H} \cdots \mathrm{O}$ in heterodimers [16] face the same problem of the dependence on many cooperative factors which is the main problem in predicting crystal structures. However, predetermination of even the simplest molecular structure is a daunting challenge because the intermolecular forces for upholding the molecules are poorly understood and difficult to predict, especially for organic solids. [17] Any step taken into predictable molecular assemblies is thereby a practical impetus toward the ultimate goal of solid-based crystal engineering.

The definition of a co-crystal has been discussed at length by various authors sometimes with conflicting opinions; [18] such a discussion will therefore not be included here, but for clarity we use the term in this work to signify a heteromeric solid form comprising neutral molecules, as opposed to salts. In this contribution, we use $1 \mathrm{H}$-benzotriazole in the co-crystallization with fumaric acid. Herein we report the crystal structure of one co-crystal compound, $\left[\left(\mathrm{C}_{6} \mathrm{H}_{5} \mathrm{~N}_{3}\right) \cdot\left(\mathrm{C}_{4} \mathrm{H}_{4} \mathrm{O}_{4}\right)_{0.5}\right]$. 


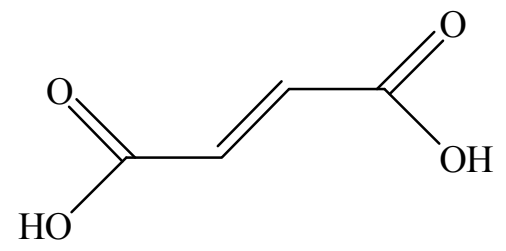

Fumaric acid

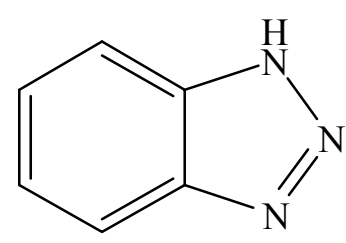

1H-Benzotriazole

Scheme 1. Chemical structures of co-formers used in this study

\section{Experimental}

\section{Materials and methods}

All chemicals used were analytical reagent and were commercially purchased. Melting point of new compound was recorded on a WRS-1B digital thermal apparatus without correction. Elemental analysis for $\mathrm{C}, \mathrm{H}$ and $\mathrm{N}$ were performed using a Perkin-Elmer 2400 elemental analyzer. IR spectrum was obtained with a Nicolet Impact 410 FTIR spectrometer using $\mathrm{KBr}$ pellets in the 4000 $\mathrm{cm}^{-1}-400 \mathrm{~cm}^{-1}$ range and was reported in the synthesis section using the following abbreviations: $\mathrm{s}=$ strong, $\mathrm{m}=$ medium, $\mathrm{w}=$ weak. A Perkin-Elmer TGA 7 thermogravimetric analyzer was used to record simultaneous TGA and DSC curves in the static $\mathrm{N}_{2}$ atmosphere at a heating rate of $10^{\circ} \mathrm{C} / \mathrm{min}$ in the temperature range $0{ }^{\circ} \mathrm{C}-800^{\circ} \mathrm{C}$ using platinum crucibles. The co-crystal was prepared as follows.

\section{Preparation of $\left(\mathrm{C}_{6} \mathrm{H}_{5} \mathrm{~N}_{3}\right) \cdot\left(\mathrm{C}_{4} \mathrm{H}_{4} \mathrm{O}_{4}\right)_{0.5}$}

A methanol $(5 \mathrm{~mL})$ solution of $1 \mathrm{H}$-benzotriazole $(90.0 \mathrm{mg}, 0.75 \mathrm{mmol})$ was added to a water solution $(5 \mathrm{~mL})$ of fumaric acid $(83.0 \mathrm{mg}, 0.50 \mathrm{mmol})$ with constant stirring for $30 \mathrm{~min}$. colorless block crystals suitable for X-ray determination were obtained after a period of 1 week upon slow evaporation of the solvents. Yield: $77 \%$, m.p.: $192^{\circ} \mathrm{C}$. Anal. Calcd (\%) for $\mathrm{C}_{8} \mathrm{H}_{7} \mathrm{~N}_{3} \mathrm{O}_{2}: \mathrm{C}, 54.23 \%$; $\mathrm{H}$, 3.98\%; N, 23.72\%. Found: C, 53.26\%; H, 4.42\%; N, 25.83\%. Infrared spectrum (KBr disc, $\left.\mathrm{cm}^{-1}\right)$ : $3235 \mathrm{~m}, 3083 \mathrm{~m}, 2920 \mathrm{~m}, 2688 \mathrm{~m}, 2535 \mathrm{~m}, 1690 \mathrm{~s}, 1595 \mathrm{~m}, 1425 \mathrm{~m}, 1387 \mathrm{~m}, 1267 \mathrm{~s}, 1217 \mathrm{~s}, 1148 \mathrm{w}$, $1089 \mathrm{w}, 1025 \mathrm{w}, 1010 \mathrm{w}, 993 \mathrm{w}, 925 \mathrm{w}, 779 \mathrm{~m}, 746 \mathrm{~m}, 646 \mathrm{~m}, 631 \mathrm{w}, 579 \mathrm{w}, 538 \mathrm{w}, 432 \mathrm{w}$.

\section{Single-crystal X-ray diffraction}

Crystals suitable for X-ray crystallography were selected using an optical microscope and glued onto a thin glass fiber. X-ray diffraction intensities for co-crystal was collected on a Siemens Smart CCD diffractometer equipped with a normal-focus, 2.4-kW sealed-tube $\mathrm{X}$-ray source (graphite-monochromatic MoKa radiation $(\lambda=0.71073 \AA)$ ) operating at $50 \mathrm{kV}$ and $40 \mathrm{~mA}$. There was no evidence of crystal decay during the data collection for the compound. The measured independent reflections were used in the structural analysis, and a semiempirical absorption correction was applied (SADABS) and the program SAINT was used for integration of the diffraction profiles [19]. The structure was solved direct methods using the SHELXS program of the SHELXTL package and refined with SHELXL [20]. The final refinement was performed by full-matrix least-squares methods with anisotropic thermal parameters for all the non-hydrogen atoms on $\mathrm{F}^{2}$. Most of the hydrogen atoms were first observed in difference electron density maps and then placed in the calculated sites and included in the final refinement in the riding model approximation with fixed thermal factors. Further details for crystallographic data and structural refinement parameters of the compound are summarized in Table 1. CCDC No. 976422.

\section{Results and Discussion}

\section{Syntheses and general characterization}

Fumaric acid and $1 \mathrm{H}$-benzotriazole both have good solubility in common organic solvents, such as $\mathrm{H}_{2} \mathrm{O}, \mathrm{CH}_{3} \mathrm{CH}_{2} \mathrm{OH}$, and $\mathrm{CH}_{3} \mathrm{OH}$. Crystallization of $1 \mathrm{H}$-benzotriazole with fumaric acid was carried out in 3:2 ratio. The complex is not hygroscopicity, and it crystallized with no solvent molecule accompanied. In the preparation of the co-crystal, the acid was mixed directly with the base in methanol and water solution, which was grown by slow evaporation of the corresponding solution at 
room temperature.

The elemental analysis data for the compound is in good agreement with its composition. The infrared spectrum of the co-crystal is consistent with its chemical formula determined by elemental analysis and further confirmed by X-ray diffraction analysis.

Table 1. Summary of the crystal data and structure refinement parameters for co-crystal

\begin{tabular}{|l|l|}
\hline Empirical formula & $\mathrm{C}_{8} \mathrm{H}_{7} \mathrm{~N}_{3} \mathrm{O}_{2}$ \\
\hline Formula weight & 177.17 \\
\hline Temperature/K & $293(2)$ \\
\hline Wavelength/ & 0.71073 \\
\hline Crystal system & monoclinic \\
\hline Space group & $P 2{ }_{1} / \mathrm{n}(14)$ \\
\hline$a / \AA$ & $11.490(2)$ \\
\hline$b / \AA$ & $4.1122(14)$ \\
\hline$c / \AA$ & $17.694(4)$ \\
\hline$\alpha /$ deg & 90 \\
\hline$\beta /$ deg & $98.575(5)$ \\
\hline$\gamma /$ deg & 90 \\
\hline Volume/ $\AA^{3}$ & $826.7(4)$ \\
\hline$Z$ & 4 \\
\hline Calculated density/g $\cdot \mathrm{cm}^{-3}$ & 1.423 \\
\hline Absorption coefficient/mm & -1 \\
\hline$F(000)$ & 0.107 \\
\hline Theta range for data collection/ ${ }^{\circ}$ & 368 \\
\hline Limiting indices & $1.99-25.65$ \\
\hline Reflections collected/unique & $-13 \leq \mathrm{h} \leq 11,-4 \leq \mathrm{k} \leq 4,-16 \leq 1 \leq 21$ \\
\hline Data/restraints/parameters & $4000 / 1500[\mathrm{R}(\mathrm{int})=0.0252]$ \\
\hline Goodness-of-fit on $F^{L}$ & $1500 / 0 / 118$ \\
\hline Final $R$ indices $[I>2 \sigma(I)]$ & 1.063 \\
\hline R indices $($ all data) & $R 1=0.0437, w R 2=0.1078$ \\
\hline Largest diff. Peak and hole/e $\cdot \AA^{-3}$ & $R 1=0.0529, w R 2=0.1150$ \\
\hline
\end{tabular}

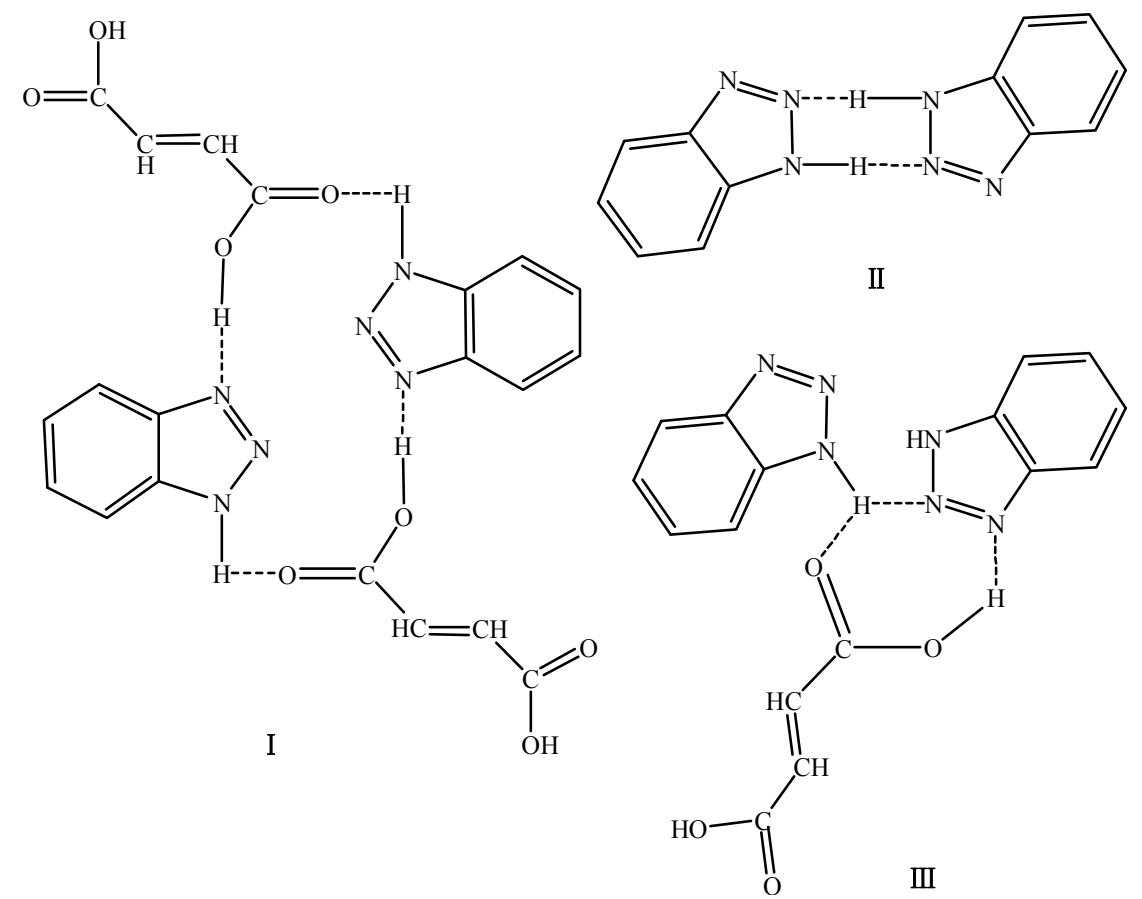

Scheme 2. Supramolecular homosynthons and heterosynthons 
Table 2. Characteristics of hydrogen-bond geometries observed in the co-crystal structure

\begin{tabular}{|c|c|c|c|c|}
\hline $\mathrm{D}-\mathrm{H} \cdots \mathrm{A}$ & $\mathrm{d}(\mathrm{D}-\mathrm{H})[\AA]$ & $\mathrm{d}(\mathrm{H} \cdots \mathrm{A})[\AA]$ & $\mathrm{D}(\mathrm{D} \cdots \mathrm{A})[\AA]$ & $\angle \mathrm{D}-\mathrm{H} \cdots \mathrm{A}\left[{ }^{\circ}\right]$ \\
\hline $\mathrm{O} 1-\mathrm{H} 1 \cdots \mathrm{N} 1 \# 1$ & 0.82 & 1.85 & $2.662(17)$ & $168.5(92)$ \\
\hline $\mathrm{N} 3-\mathrm{H} 3 \cdots \mathrm{O} 2 \# 2$ & 0.86 & 2.07 & $2.888(19)$ & $157.4(93)$ \\
\hline $\mathrm{N} 3-\mathrm{H} 3 \cdots \mathrm{N} 2 \# 1$ & 0.86 & 2.53 & $2.953(21)$ & $111.0(96)$ \\
\hline $\mathrm{C} 3-\mathrm{H} 6 \cdots \mathrm{O} 1 \# 3$ & 0.93 & 2.69 & $3.342(24)$ & $127.5(115)$ \\
\hline
\end{tabular}

Symmetry transformations used to generate equivalent atoms: \#1 -1+x, y, z; \#2 x, y, z; \#3 x, y, -1+z.

(a)

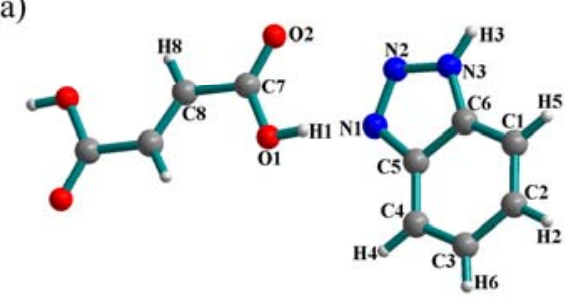

(b)

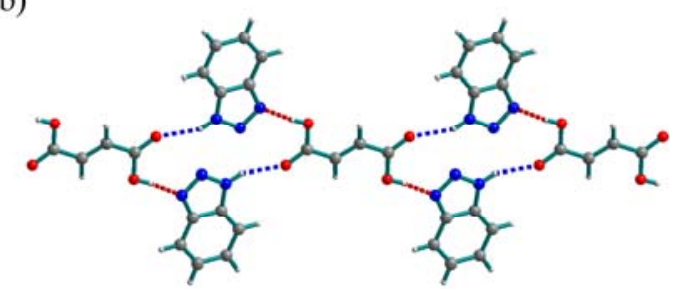

(c)

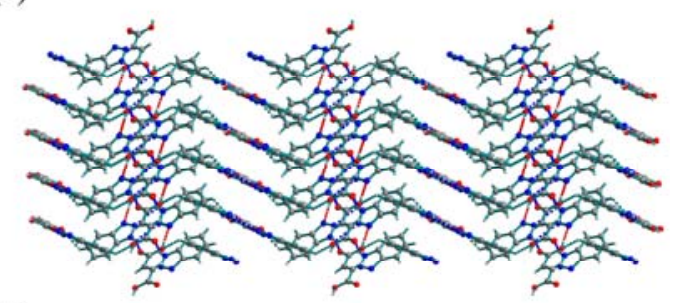

(d)

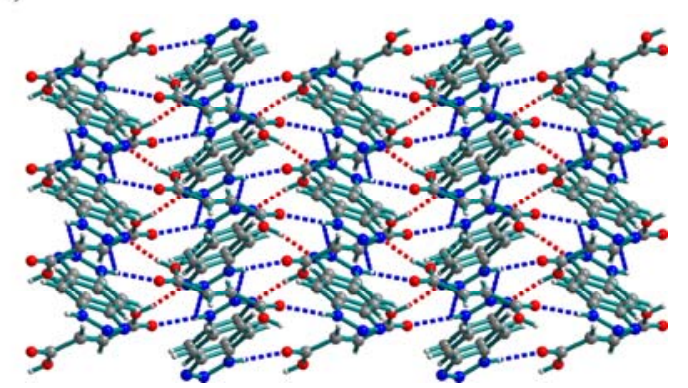

Figure 1. (a) Molecular structure with atom labeling of the asymmetric unit; (b) 1D chain array via hydrogen bonds along the [010] direction (the hydrogen bonds are indicated as broken lines in this and the subsequent figures); (c) and (d) 3D hydrogen-bonded supramolecular structure. (O, red; N, blue; C, gray; H, light gray in

\section{Crystal structure of the co-crystal} this)

The schematic representations of supramolecular synthons related to this work are summarized in Scheme 2. Possible hydrogen-bonding geometries are listed in Table 2. Single crystals of co-crystal were obtained by dissolving fumaric acid and $1 \mathrm{H}$-benzotriazole $(3: 2)$ in a methanol and water solvent mixture. The single crystal structure reveals that it is crystallized in a monoclinic space group, $P 2{ }_{1} / \mathrm{n}(14)$. The fumaric acid molecule lies on an inversion center with only half the molecule contained in the crystallographic asymmetric unit, along with a whole $1 \mathrm{H}$-benzotriazole molecule (Figure. 1a). The distances for $\mathrm{COOH}(\mathrm{O} 1-\mathrm{C} 7,1.311 \AA)$ support the existence of nonionic acid moieties indicating co-crystal formation. The exocyclic bond length C7-C8 is elongated to $1.484 \AA$, longer than the cyclic those of C-C bonds (average 1.388 $\AA$ ). The bond lengths $\mathrm{C}-\mathrm{N}$ are average 1.367 
$\AA$, and the bond lengths N-N are average $1.318 \AA$. The fumaric acid molecule resides an inversion center conformation with the $\mathrm{C} 7-\mathrm{C} 7-\mathrm{C} 8-\mathrm{C} 7$ twist angle of $180.0(15)^{\circ}$. As illustrated in Figure 1a, the local structure of co-crystal consists of base and acid molecules. Within 1H-benzotriazole molecule moiety, the dihedral angle between phenyl ring and triazole plane is $1.6(168)^{\circ}$ indicating the coplanarity of both rings. The triazole plane and phenyl ring plane (both sitting on fumaric acid molecule) form dihedral angles of $4.0(142)^{\circ}$ and $3.9(176)^{\circ}$ with the carboxyl group plane $(\mathrm{C} 7 / \mathrm{O} 1 / \mathrm{O} 2)$ of the fumaric acid molecule, respectively. Fumaric acid molecules interact with $1 \mathrm{H}$-benzotriazole molecules via $\mathrm{O} 1-\mathrm{H} 1 \cdots \mathrm{N} 1$ and $\mathrm{N} 3-\mathrm{H} 3 \cdots \mathrm{O} 2$ hydrogen bonds with rings to form a linear infinite chain running parallel to the b-axis as shown in Figure 1b. The graph set representation of this ring is $R_{4}^{4}(16)$ (synthon I ). As a consequence, the alternate combination of such a ring network is spread over the whole space, and each connects the adjacent tape via N3-H3 $\cdots \mathrm{N} 2$ and $\mathrm{C} 3-\mathrm{H} 6 \cdots \mathrm{O} 1$ hydrogen bonds (homosynthon II $R_{2}^{2}(6)$ and heterosynthon III $R_{2}^{3}(7)$ ), which account for the formation of a 3D supramolecular assembly as shown in Figure 1c. These networks adopt a parallel stack along [001] with an offset, as depicted in Figure 1d, and no interlayer interactions such as hydrogen bonding or aromatic stacking are observed.

\section{Thermal analysis}

The thermal stability of the compound previously presented was assessed by combining data from different techniques such as DTA and TGA.

In the DTA thermogram, the melting point is observed at $218^{\circ} \mathrm{C}$, immediately followed by decomposition. The melting point of the co-crystal shows that it melts at higher temperature that $1 \mathrm{H}$-benzotriazole $\left(98^{\circ} \mathrm{C}\right)$ but lower than fumaric acid $\left(299^{\circ} \mathrm{C}\right)$. This is probably the result of the closer molecular packing in the crystal structure, and the intermolecular hydrogen bonding also affects the melting point of the compound.

The thermal analysis under nitrogen atmosphere shows that co-crystal begins to decompose with melting at $26^{\circ} \mathrm{C}$ and instantly decomposes after melting. The co-crystal undergoes decomposition in two stages. The first stage from $26^{\circ} \mathrm{C}$ to $101{ }^{\circ} \mathrm{C}$ corresponds to degradation of fumaric acid molecule with weight loss of $13.75 \%$. The second stage occurs from $101^{\circ} \mathrm{C}$ to $590^{\circ} \mathrm{C}$ with a mass loss of $81.6 \%$ that correlate with the decomposition of $1 \mathrm{H}$-benzotriazole molecule.

\section{Conclusions}

Organic solid based on fumaric acid and 1H-benzotriazole has been prepared and characterized by chemical techniques, which exhibit multiform supramolecular synthons due to the participation of hydrogen-bonded donors and acceptors with different intensities. Single crystal structure of the co-crystal was determined in order to elucidate the supramolecular hydrogen bonded network in such solid. The double bond in fumaric acid as spacer between two carboxy groups leads to a planar arrangement of $1 \mathrm{H}$-benzotriazole and fumaric acid molecules. The fumaric acid molecules and 1H-benzotriazole molecules act as to link the infinite chains via the expected carboxyl-azole heterosynthons, and form three-dimensional supramolecular network with $\mathrm{N}-\mathrm{H} \cdots \mathrm{N}$ and weak $\mathrm{C}-\mathrm{H} \cdots \mathrm{O}$ type hydrogen bonding interactions are present in the co-crystal. The formation of $\mathrm{O}-\mathrm{H} \cdots \mathrm{N}$ and $\mathrm{N}-\mathrm{H} \cdots \mathrm{O}$ hydrogen bonds in supramolecular complexes of N-heterocyclic base and carboxylic acid is generally predictable, but weak $\mathrm{C}-\mathrm{H} \cdots \mathrm{O}$ interactions may be affected by geometrical compatibility between base and acid molecules. Co-crystal has melting point which was between the starting materials. Further studies on such flexible building block (fumaric acid) are desirable to understand the crystal engineering of hydrogen-bonded solids, and related exploration on this perspective is underway.

\section{Acknowledgement}

In this paper, the research was sponsored by the National Natural Science Foundation of China (No. 21203106 and 51372125), the Natural Science Foundation of Shandong Province, China ((No. ZR2011BL015), and the State Key Laboratory of Inorganic Synthesis and Preparative Chemistry of 
Jilin University (No. 2013-34).

\section{References}

[1] Yang W., Greenaway A., Lin X., et al, J. Am. Chem. Soc. 2010, 132,14457

[2] Aakeröy C. B., Desper J., Scott B. M. T, Chem. Commun. 2006, 1445

[3] Bhogala B. R. , Nangia A., New J. Chem. 2008, 32, 800

[4] Wang L., Zhao L., Xu L. Y., CrystEngComm. 2013, 15, 2835

[5] Wang L., Zhao L., Xue R. Y., Sci. China. Chem. 2012, 55, 2515

[6] Chiarella R. A., Davey R. J., Peterson M. L., Cryst. Growth Des. 2007, 7: 1223

[7] Oliveira M. A., Peterson M. L., Klein D., Cryst. Growth Des. 2008, 8, 4487

[8] (Nichol G. S., Clegg W., Cryst. Growth Des. 2009, 8, 1844

[9] Baburin I. A., Blatov V.A., Carlucci L., Cryst. Growth Des. 2008, 8: 519

[10]Braga D., Grepioni F., New J. Chem. 1998, 11, 1159

[11]Calhorda M. J., Chem. Commun. 2000, 801

[12]Braga D., Grepioni F., New J. Chem. 1998, 11, 1159

[13]Desiraju G. R., Steiner T., Oxford University Press, 1999: Oxford

[14]Batchelor E., Klinowski J., Jones W.J., Mater. Chem. 2000, 10, 839

[15] Senthil V. S., Nangia A., Katz A. K., et al, Cryst. Growth Des. 2002, 2, 313

[16]Wang L., Zhao L., Liu M., Sci. China. Chem. 2012, 55, 2115

[17] Sarma J. A. R. P., Desiraju G. R., Cryst. Growth Des. 2002, 2, 93

[18]SMART and SAINT; Siemens Analytical X-ray Instruments, Inc.: Madison, W I, 1996.

[19] SHELXTL,Version 5.1; Siemens Industrial Automation. Inc., 1997. 